\title{
Remote Sensing and GIS as an Advance Space Technologies for Rare Vegetation Monitoring in Gobustan State National Park, Azerbaijan
}

\author{
Yelena M. Gambarova ${ }^{1}$, Adil Y. Gambarov ${ }^{2}$, Rustam B. Rustamov ${ }^{3}$, Maral H. Zeynalova ${ }^{4}$ \\ ${ }^{1}$ R.I.S.K. Company, Baku, Azerbaijan \\ ${ }^{2}$ SAHIL IT Company, Baku, Azerbaijan \\ ${ }^{3}$ Institute of Physics of the National Academy of Sciences, Baku, Azerbaijan \\ ${ }^{4}$ Institute of Botany of the National Academy of Sciences, Baku, Azerbaijan \\ E-mail: elenag@risk.az,AGambarov@sahil.info
}

\begin{abstract}
This paper describes remote sensing methodologies for monitoring rare vegetation with special emphasis on the Image Statistic Analysis for set of training samples and classification. At first 5 types of Rare Vegetation communities were defined and the Initial classification scheme was designed on that base. After preliminary Statistic Analysis for training samples, a modification algorithm of the classification scheme was defined: one led us to creating a 4 class's scheme (Final classification scheme). The different methods analysis such as signature statistics, signature separability and scatter plots are used. According to the results, the average separability (Transformed Divergence) is 1951.14 , minimum is 1732.44 and maximum is 2000 which shows an acceptable level of accuracy. Contingency Matrix computed on the results of the training on Final classification scheme achieves better results, in terms of overall accuracy, than the training on Initial classification scheme.
\end{abstract}

Keywords: Remote Sensing, GIS, Seperability, Classification

\section{Introduction}

The vegetation is one of the key and best instrument and indicator for monitoring of identification of impacts of the natural processes, environmental and ecological issues. As changes in vegetation are rapid and serious due to various human activities, it is urgent to monitor vegetation and their surrounding environment from physical, biological or social viewpoints. Remote sensing is expected to provide us an efficient tool for monitoring vegetation environment. In particular, as considering vegetation is often characterized by a mixture of different vegetations, soil and water, remote sensing is expected to delineate the relation between them.

This paper describes Remote Sensing and GIS as an advance Space Technology for Rare Vegetation monitoring in Gobustan State National Park with special emphasis on Image Statistic Analysis for set of training samples and classification.

Determination of the 'best' bands combinations in the context of Image statistical analysis is very important.
The best band combinations will be used in accurate classification. Methods used to select the optimum bands combination are known as feature selection techniques.

A number of criteria can be used to categorize feature selection techniques. As they can be classified on the basis of whether they are graphical or statistical in nature [1], they can also be classified into two categories based on whether or not they use classification algorithms to evaluate the performance of subsets. Techniques that use the former approach are called 'wrapper techniques'; techniques using the latter approach are known as 'filter techniques' [2].

A filter is defined as a feature selection algorithm using a performance metric based entirely on the training data, without reference to the classifier for which the features are to be selected. The most widely used filter methods are based on class separability indices. Use of this approach in the context of Image statistical analysis was investigated in this study. Class Separability indices were employed to determine the best band combination of SPOT 5 image datasets. 
These indices have been extensively used by researchers in remote sensing for many years [3-5].

Some researchers sought to test whether some bands had more discriminating power than others by using the Jeffries-Matusita distance analysis technique only [3], [5] and [6]. Other researchers, for this purpose, Divergence Distance or Battacharrya Distance were used to measure the separability [4], [5] and [7].

In our case, of the four separability indices compared, the use of transformed measures (Transformed Divergence and the Jeffries-Matusita distance) in the Class Separability appeared to be more powerful than other methods. Transformed divergence and the Jeffries-Matusita distance both found the best solution with the highest classification accuracy.

\section{Study Area}

This study was carried out in Gobustan, located between the southern outcrops of the Caucasus Mountain range and the Caspian Sea, some $60 \mathrm{~km}$ south of the capital Baku as in presented in the Figure 1.

The Study Area at Gobustan (covering the area of $282 \mathrm{~km}^{2}$ ) contains a wealth of historical and archaeological sites and is also known for its rare vegetation.

The vegetation communities in the study area repressent the most ecologically important habitat. Some of Rare Vegetation communities within the expected for investigation area presently classified as either rare or threatened and recommended for inclusion into the National Red Book. The importance of this habitat type is one of the reasons why the Gobustan State National Park has been proposed, so that some level of protection is offered to this desert.

\section{Data Used and Methodology}

Four SPOT5 images in $2.5 \mathrm{~m}$ and $5 \mathrm{~m}$ resolutions, acquired between 2004 and 2007 were used for the delineation and classification of Rare Vegetation communities.

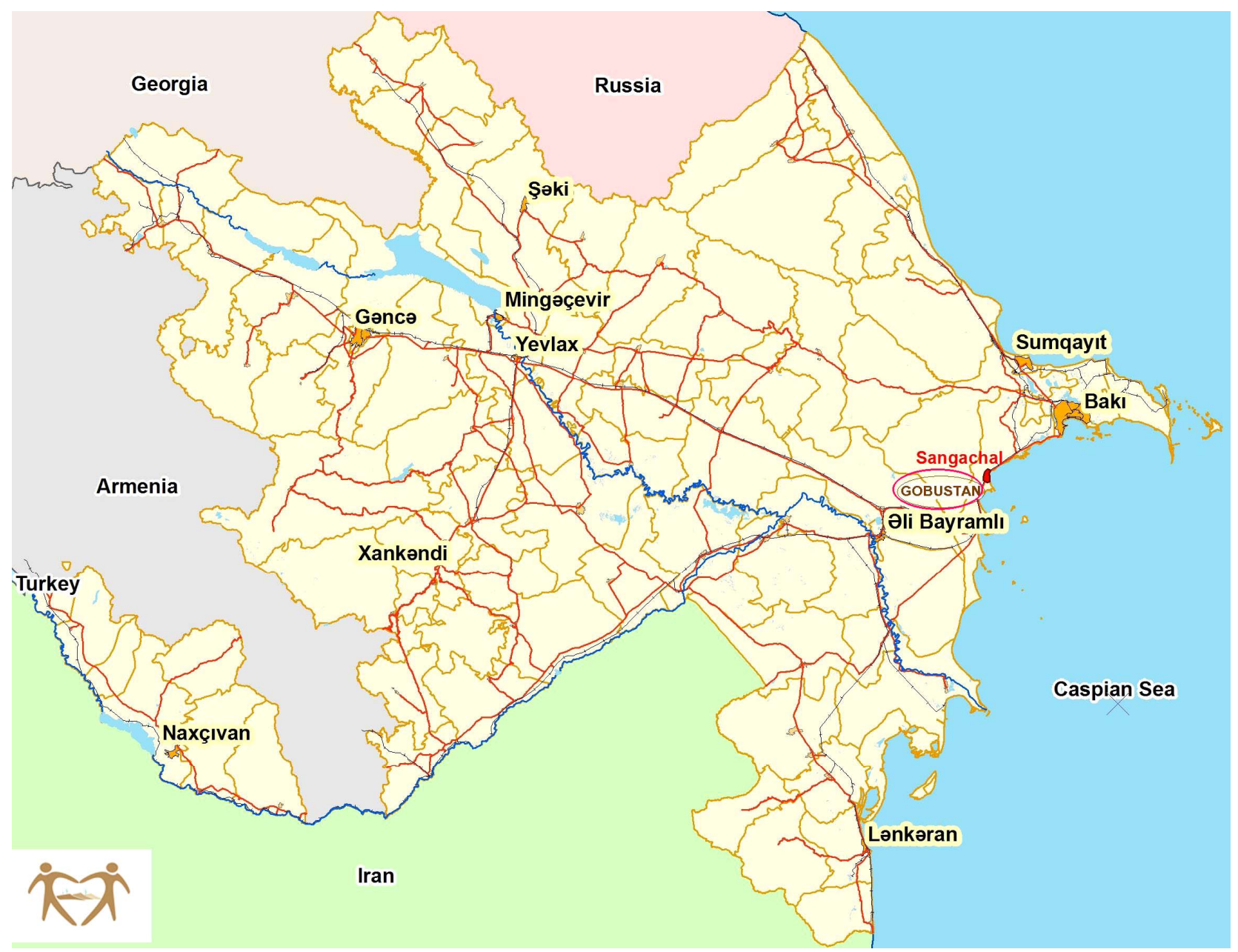

Figure 1. Study area. 
The sampling scheme was designed to collect the rare vegetation communities in Gobustan National Park study site for combined ecological and remote sensing studies. The Field surveys were hold in accordance with preliminary data on the spreading of rare plants in the study area. Quadrates and plots assisted by satellite SPOT 5 imagery have provided information on habitat types and status. Because GPS devices provided the coordinates for groundreference data during fieldwork, the sample plots were accurately linked to SPOT imagery. Every plot was registered with GPS Garmin device to allow further integration with spatial data in GIS and image processing systems (Figure 2).

\section{Definition of the Initial Classification Scheme}

Classification process involves three steps: 1) training, 2) classification and 3) output and validation.

In the training stage Initially 5 types of Rare Vegetation communities were defined that-according to ecologists' opinion-are indicators of climate and ecosystem properties in the region being studied. Below the Latin names of them are presented (Table 1). At first, these sites were geolocated, then using GIS procedures the areas of location of these vegetation communities were determined for extraction of samples for the classifier training and testing.

The set of training samples was tested for Representativeness and Separability based on their calculated statistical parameters. There are the tests to perform that can help determine whether the set of training samples are a true representation of the pixels to be classified for each class.

It is important that the training areas be representative of the full variability of spectral response in that class. Author [8] recommends that a minimum of $10 \mathrm{n}$ to $100 \mathrm{n}$ pixels be part of training areas, where $n$ is the number of spectral bands. Hence, in our case, with SPOT data, the requirement is for roughly 30 to 300 pixels per class.

\subsection{Image Statistical Analysis: The Initial Classification Scheme}

Once the training areas are selected, different methods are used for testing purposes such as histograms, separability, signature statistics and scatter plots.

The visualization technique in feature space allows estimating range of the correlation of training samples: thereto, for each of the class from the training data was estimated of Minimum and Maximum values on each band used and created three-dimensional parallelepiped in the feature space. Or, another way is to define a threedimensional ellipsoid, estimated of Mean \pm Standard deviations values on each band used.

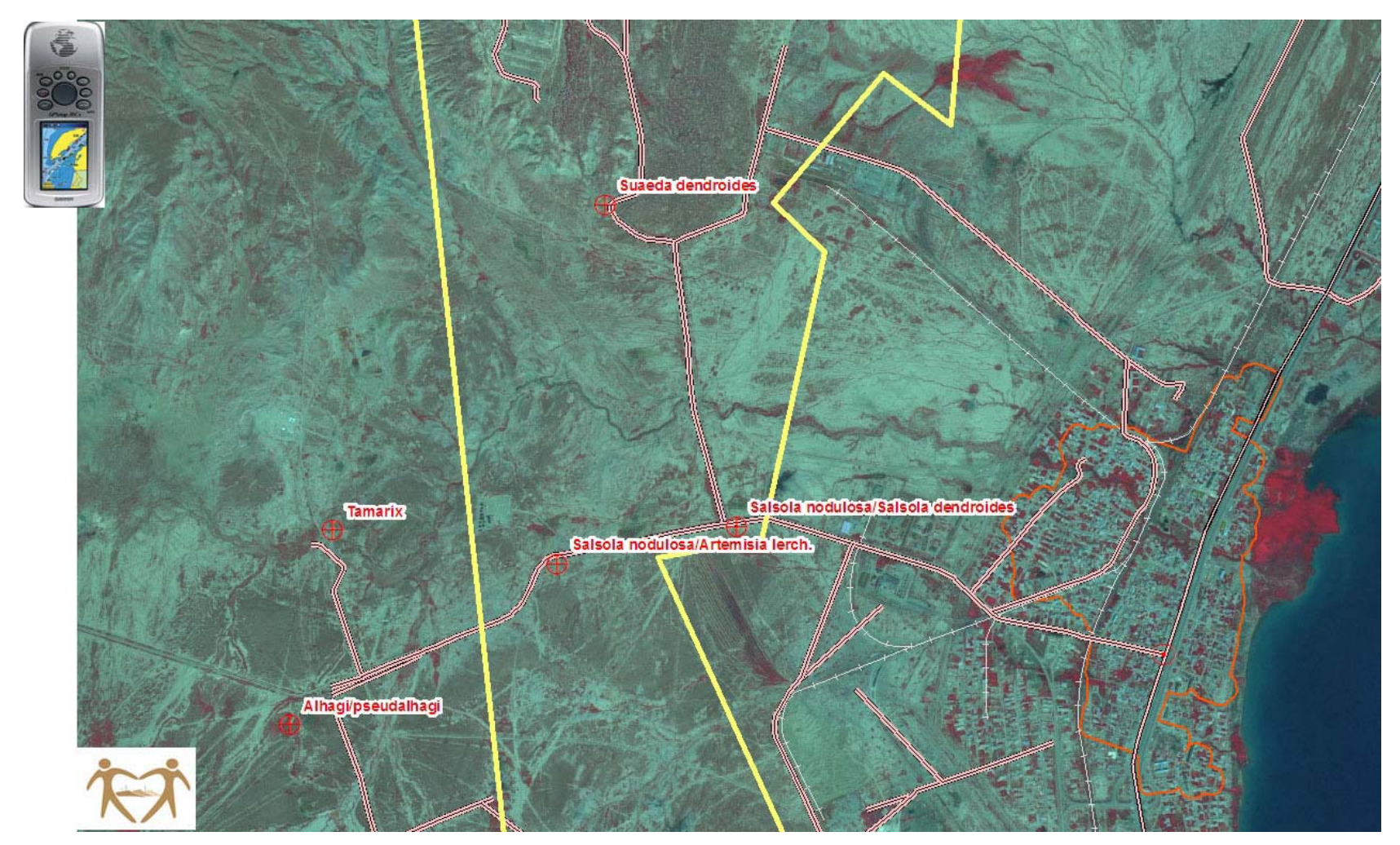

Figure 2. Interpretation of SPOT image and field survey. 
Table 1. Rare vegetation communities. Initial classification scheme.

\begin{tabular}{cl}
\hline Class & The name of vegetation communities \\
\hline Class 1 & Alhagi pseudoalhagi \\
Class 2 & Salsola Nodulosa/Artemisia Lerchiana \\
Class 3 & Salsola Nodulosa/Salsola Dendroides \\
Class 4 & Tamarix \\
Class 5 & Suaeda Dendroides \\
\hline
\end{tabular}

\subsection{Compare Ellipses}

We can view graphs of these statistics for compare classes. The graphs display as sets of ellipses in a Feature Space image. Each ellipse is based on the mean and standard deviation of one class. The color is used as the color for the class in the visualization functions, ellipses, etc. The ellipses are presented with the color regarding each class as is shown in table below.

\begin{tabular}{cl|l}
\hline Class Number & The name of vegetation communities & Color \\
\hline Class 1 & Alhagi pseudoalhagi & \\
Class 2 & Salsola Nodulosa/Artemisia Lerchiana & \\
Class 3 & Salsola Nodulosa/Salsola Dendroides & \\
Class 4 & Tamarix & \\
Class 5 & Suaeda Dendroides & \\
\hline
\end{tabular}

By comparing the ellipses for different classes for a one band pair, it is easy to see if the training set represents similar groups of pixels by seeing where the ellipses overlap on the Feature Space image (Figure 3). As shown in Figure 3, the ellipses are overlapped, that means the set of training samples (excepting class Alhagi pseudoalhagi) represent similar pixels, which is not desirable for classification.

\subsection{Class Separability: Initial Classification Scheme}

Separability can be evaluated for any combination of bands that is used in the classification, enabling you to rule out any bands that are not useful in the results of the classification. These distances used to determine the best results to use in the classification. If the spectral distance between two samples is not significant for any pair of bands, then signatures may not be distinct enough to produce a successful classification. We evaluated the Average and Minimum Separability on all formulas for the band set. The Best Minimum and Best Average Separability values present in Table 2.
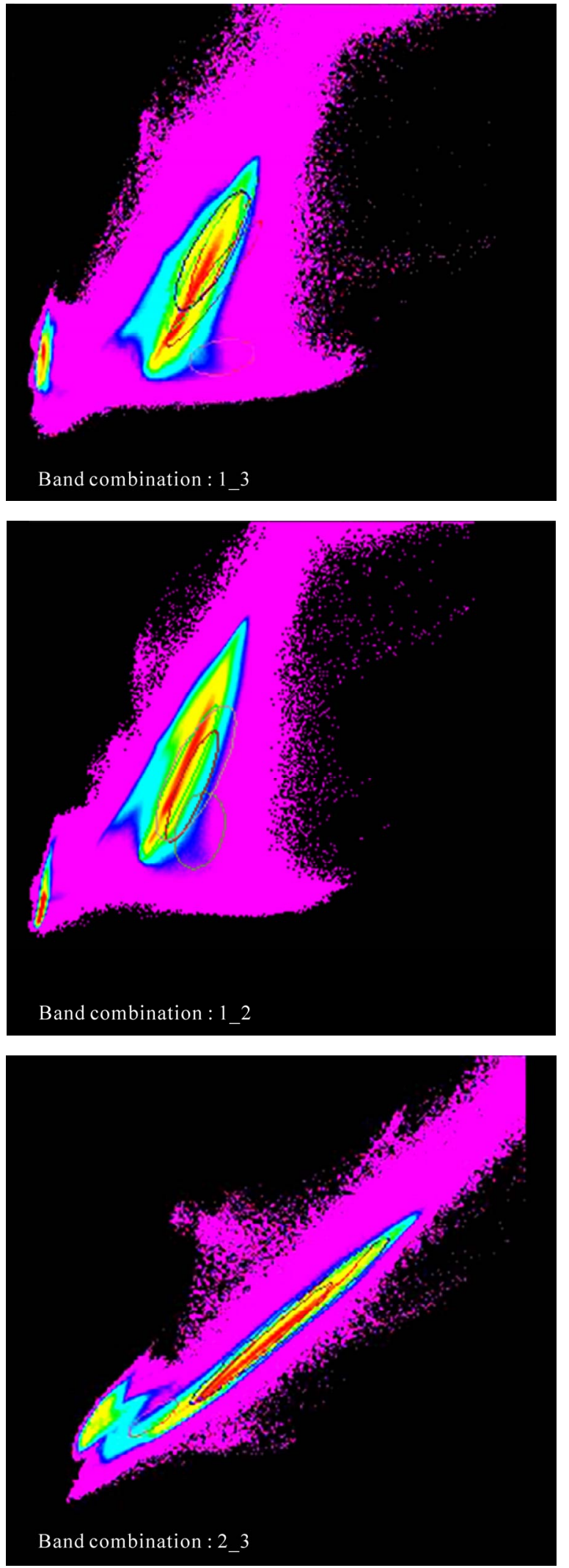

Figure 3. Band combination. Sets of ellipses in the feature space image. 
Although for completeness we presented all four methods for calculating separability (Table 3), generally two different formulas were used: Transformed Divergence (TD) and Jeffries-Matusita distance (JM).

Transformed Divergence and the Jefferies-Matusita distance both found the best solution with the highest classification accuracy.

According [1] both TD and JM have upper and lower bounds:

Transformed Divergence is between 0 and 2000

Jefferies-Matusita Distance is between 0 and 1414

As a general rule, if the result is greater than 1900, then classes can be separated. Between 1700 and 1900 the separation is fairly good. Below 1700 , the separation is poor [1].

Analyzing the results shown in Table 2 we can unambiguously concluded that the classes are poor separable (Class separability values greatly lower bounds) and these training samples could not used for accuracy classification. For confirmation this conclusion a Contingency Matrix was calculated (Table 3).

\subsection{Contingency Matrix: Initial Classification Scheme}

Contingency Matrix do a quick classification of the pixels in a set of training samples to see what percentage of the sample pixels are actually classified as expected [9].
In theory, each training sample would be composed primarily of pixels that belong to its corresponding class. Practically, as are shown in Table 4, only all pixels from Class 1-Alhagi pseudoalhag — classified correctly (assigned to its class). The overall accuracy was calculated by summing the main diagonal elements of the Contingency matrix and dividing by the total number of samples.

These tests have shown that: Class 3 has completely contained Class 2; Class 4 and Class 5 have heavily overlapped each other. These undesirable results of Statistical tests and Class Separability generated the need to perform any operations to improve (qualify) of Initial classification scheme. These tests pointed out to a direction of possible modification of "Initial classification scheme", for that an additional set of training samples was required.

\section{Definition of the Final Classification Scheme}

In the during field surveys a new sites for collection training samples was defined.

After analyzing the results it would be beneficial to merge Class 2 (Salsola Nodulosa/Artemisia Lerchiana and Class 3 (Salsola Nodulosa/Salsola Dendroides) into one class:

Table 2. Best minimum and best average separability (Initial classification scheme).

\begin{tabular}{|c|c|c|c|c|c|c|c|c|c|c|}
\hline \multicolumn{3}{|c|}{ Band Combination } & \multicolumn{2}{|c|}{ Euclidean Distance } & \multicolumn{2}{|c|}{ Divergence } & \multicolumn{2}{|c|}{ Transformed Divergence } & \multicolumn{2}{|c|}{ Jefferies-Matusita Distance } \\
\hline & & & MIN & AVE & MIN & AVE & MIN & AVE & MIN & AVE \\
\hline & 2 & 3 & 3 & 36 & 1 & 99 & 286 & 1414 & 525 & 1088 \\
\hline 1 & 2 & & 3 & 29 & 2 & 463 & 527 & 1730 & 436 & 1043 \\
\hline 1 & 3 & & & & 4 & 425 & 787 & 1699 & 408 & 1052 \\
\hline 2 & 3 & & 3 & 35 & & & & & & \\
\hline 2 & & & 2 & 29 & 4 & 472 & 747 & 1736 & 123 & 741 \\
\hline
\end{tabular}

Table 3. Contingency matrix. Initial classification scheme.

\begin{tabular}{|c|c|c|c|c|c|c|}
\hline Class Number & Class 1 & Class 2 & Class 3 & Class 4 & Class 5 & Row Total \\
\hline Class 1 & 226 & 0 & 0 & 0 & 0 & 226 \\
\hline Class 2 & 0 & 775 & 212 & 205 & 11 & 1203 \\
\hline Class 3 & 0 & 88 & 644 & 126 & 2 & 860 \\
\hline Class 4 & 0 & 352 & 378 & 471 & 0 & 1201 \\
\hline Class 5 & 0 & 169 & 115 & 24 & 176 & 484 \\
\hline Column Total & 226 & 1384 & 1349 & 826 & 189 & 3974 \\
\hline \multicolumn{7}{|c|}{ Overall Accuracy $=57.6 \%$} \\
\hline
\end{tabular}


(SalsolaNodulosa/ArtemisiaLerchiana_SalsolaNodulosa/ SalsolaDendroides).

The algorithm of this modification is presented (Table 4). There was received the Final classification scheme consisted of four classes:

Having received the new set, we performed the same statistical tests of representativeness and separability which show the advances have come using new Final classification scheme.

\subsection{Class Separability: Final Classification Scheme}

The Class Separability on Final classification scheme was arranged in matrix form.

We evaluated Transformed Divergence (TD) and Jefferies-Matusita Distance (JM) for every class pair and one band combination. Then we compared these num- bers (values) to other separability listings for other band combinations to determine which set of bands is the most useful for classification.

The Table 5 and Table 6 present the Transformed Divergence matrix and the Jefferies-Matusita Distance separability matrix on the best band combinations.

Analyzing the numerical $T D$ values (Table 5) we can conclude that the separability results for training samples on final classification scheme are good enough with the exception of class pair 2:4. The Best Average Separability is 1951.14, Minimum Separability is 1732.44 and Maximum Separability is $\mathbf{2 0 0 0}$. That is to say Class Separability values greater than 1900 where obtained for most classes, besides for Class 1 the TD value is $\mathbf{2 0 0 0}$ - upper bound.

Also the values of the $J M$ distance for the data set (Table 6) are greater than the values obtained from Initial scheme data (Table 2). Having acceptable levels for the separability of the training areas, the next step is to conduct the classification process.

Overall, Class Separability is adequate and would provide a fairly accurate classification.

Table 4. Final classification scheme.

\begin{tabular}{cl}
\hline Class & \multicolumn{1}{c}{ Classified Data } \\
\hline Class 1 & Alhagi pseudoalhagi \\
Class 2 & $\begin{array}{l}\text { Salsola Nodulosa/Artemisia Lerchiana_Salsola } \\
\text { Nodulosa/Salsola Dendroides }\end{array}$ \\
Class 3 & Tamarix \\
Class 4 & Suaeda Dendroides \\
\hline
\end{tabular}

Table 5. Transformed divergence separability matrix for training classes.

Distance Measure: Transformed Divergence

Best Average Separability: 1951.14

Band Combination: 1_2

\begin{tabular}{lcccc}
\multicolumn{1}{c}{ Signature Name } & Class & 1 & 2 & 3 \\
\hline Alhagi pseudoalhagi & 1 & 0 & 2000 & 0 \\
Tamarix & 2 & 2000 & 1975.13 & 0 \\
Suaeda Dendroides & 3 & 2000 & 1975.13 & 132.44 \\
$\begin{array}{l}\text { Salsola Nodulosa/Artemisia Lerchiana_Salsola } \\
\text { Nodulosa/Salsola Dendroides }\end{array}$ & 4 & 2000 & 1732.44 \\
\hline
\end{tabular}

Table 6. Jefferies-matusita distance separability matrix for training classes.

Distance Measure: Jefferies-Matusita

Best Average Separability: 1208.63

Band Combination: 1_2_3

\begin{tabular}{|c|c|c|c|c|c|}
\hline Signature Name & Class & 1 & 2 & 3 & 4 \\
\hline Alhagi pseudoalhagi & 1 & 0 & 1411.5 & 1402.88 & 1367.45 \\
\hline Tamarix & 2 & 1411.5 & 0 & 1255.07 & 1010.43 \\
\hline Suaeda Dendroides & 3 & 1402.88 & 1255.07 & 0 & 904.43 \\
\hline $\begin{array}{l}\text { Salsola Nodulosa/Artemisia Lerchiana_Salsola } \\
\text { Nodulosa/Salsola Dendroides }\end{array}$ & 4 & 1367.45 & 1010.43 & 904.43 & 0 \\
\hline
\end{tabular}


Table 7. Contingency matrix. Final classification scheme.

\begin{tabular}{|c|c|c|c|c|}
\hline Classified Data & Alhagi pseudoalhagi & Tamarix & Suaeda Dendroides & $\begin{array}{l}\text { SalsolaNodulosa/Artemisia } \\
\text { Lerchiana/SalsolaNodulosa/Salsola } \\
\text { Dendroides }\end{array}$ \\
\hline Alhagi pseudoalhagi & 151 & 0 & 0 & 28 \\
\hline Tamarix & 0 & 342 & 0 & 151 \\
\hline Suaeda Dendroides & 1 & 11 & 65 & 128 \\
\hline $\begin{array}{l}\text { SalsolaNodulosa/Artemisia } \\
\text { Lerchiana_SalsolaNodulosa/Salsola } \\
\text { Dendroides }\end{array}$ & 5 & 20 & 11 & 462 \\
\hline Column Total & 157 & 373 & 76 & 769 \\
\hline Overall Accuracy = 74.2\% & & & & \\
\hline
\end{tabular}

\subsection{Contingency Matrix: Final Classification Scheme}

A common method for classification accuracy assessment is through the use of the Contingency Matrix. The Overall Accuracy is 74.2\% (Table 7).

It has been found that the Contingency Matrix computed on the results of the training on Final classification scheme achieves better results, in terms of overall accuracy (overall accuracy $=74.2 \%$ ) than the training on Initial classification scheme (overall accuracy $=57.6 \%$ ).

\section{Conclusions}

The aim of this study was to perform the Image Statistical analysis in the training stage. The number of multivariate statistical techniques was employed to estimate the degree of discrimination between the classes. At every step of the training process, values of Class Separability as represented by Transformed Divergence and Jefferies-Matusita Distance where evaluated as a measure of the quality of training areas. Training areas for first dataset (Initial classification scheme) that produced $T D$ coefficients lower than 1700 for either measure where rejected (Table 2 and Table 3 ).

The Image Statistical analysis of Final classification scheme (modified scheme) have shown the advances of new Final classification scheme and determined the best combinations of bands for separating the classes from each other (Table 6 and Table 7).

The accuracy in this classification suggested that this strategy for the selection of training samples, modification of classification scheme used were importance to perform better classification result.

\section{Acknowledgements}

This work was supported by the Planet Action and the
Idea Wild non-profit organizations for their support by donating satellite images, GIS software and equipment, which provided recourses for the research that led to this paper.

\section{References}

[1] J. R. Jensen, "Introductory Digital Image Processing: A Remote Sensing Perspective,” Prentice Hall, London, 1996.

[2] T. Kavzoglu and P. Mather, "The Role of Feature Selection in Artificial Neural Network Applications,” International Journal Remote Sensing, Vol. 23, No. 15, 2002, pp. 2919- 2937.

[3] I. L. Thomas, V. M. Benning and N. P. Ching, "Classification of Remotely Sensed Images,” Adam Hilger, London, 1987.

[4] L. V. Dutra and R. I. Huber, "Feature Extraction and Selection for ERS-1/2 in SAR Classification,” International Journal of Remote Sensing, Vol. 20, No. 5, 1999, pp. 993-1016.

[5] B. M. Tso and P. M. Mather, "Crop Discrimination Using Multi-Temporal SAR Imagery,” International Journal of Remote Sensing, Vol. 20, No. 12, 1999, pp. 2443-2460.

[6] O. Mutanga, I. Riyad, A. Fethi and K. Lalit, "Imaging Spectroscopy (Hyperspectral Remote Sensing) in Southern Africa: An Overview," South African Journal of Science, Vol. 105, No. 5-6, 2009, pp. 83-96.

[7] H. I. Mohd and J. Kamaruzaman, "Satellite Data Classification Accuracy Assessment Based from Reference Dataset," International Journal of Computer and Information Science and Engineering, 2008, pp. 96-102.

[8] T. M. Lillesand, R. W. Kiefer and J. W. Chirman, "Remote Sensing and Image Interpretation,” Wiley, Hoboken, 2004.

[9] P. C. Smits, S. G. Dellepiane and R. A. Schowengerdt, "Quality Assessment of Image Classification Algorithms for Land-Cover Mapping: A Review and a Proposal for a Cost-Based Approach,” International Journal of Remote Sensing, Vol. 20, No. 8, 1999, pp. 1461-1486. 